\title{
ON THE DIFFERENTIAL EQUATIONS OF H. LEWY
}

\author{
W. B. SMITH-WHITE
}

(received 10 October 1960)

\section{Introduction}

It is known that the theory of Cauchy's problem for differential equations with two independent variables is reducible to the corresponding problem for systems of quasi-linear equations. The reduction is carried further, by means of the theory of characteristics, to the case of systems of equations of the special form first considered by $\mathrm{H}$. Lewy [1]. The simplest case is that of the pair of equations

$$
\left.\begin{array}{l}
a_{11} \frac{\partial z_{1}}{\partial x}+a_{12} \frac{\partial z_{2}}{\partial x}=0 \\
a_{21} \frac{\partial z_{1}}{\partial y}+a_{22} \frac{\partial z_{2}}{\partial y}=0,
\end{array}\right\}
$$

where the $a_{i j}$ depend on $z_{1}$ and $z_{2}$. The problem to be considered is that of finding functions $z_{1}(x, y), z_{2}(x, y)$ which satisfy (1) and which take prescribed values on $x+y=0$.

For brevity we shall say that a function $f(a, b, c, \cdots)$ of the arguments $a, b, c, \cdots$ is of class $C^{(n)}[a, b, c, \cdots]$, or simply of class $C^{(n)}$, when all the partial derivatives of $f$ with respect to these variables of order $\leqq n$ exist and are continuous. If on the line $x=\lambda, y=-\lambda$ the Cauchy data are $C^{(2)}[\lambda]$, if the coefficients $a_{i j}$ are $C^{(2)}\left[z_{1}, z_{2}\right]$, and if

Lewy showed

$$
\Delta=\left|\begin{array}{ll}
a_{11} & a_{12} \\
a_{21} & a_{22}
\end{array}\right| \neq 0,
$$

(I) that there exists a solution-pair $z_{1}, z_{2}$ of equations (1) of class $C^{(1)}[x, y]$ defined near $x=y=0$ and which takes the prescribed values on $x+y=0$, and for which the "mixed" derivatives

$$
\frac{\partial^{2} z_{1}}{\partial x \partial y}, \frac{\partial^{2} z_{2}}{\partial x \partial y}
$$

exist and are continuous. 
(II) that the solution just described is unique.

Lewy's method consists in replacing the differential equations by difference equations and then establishing the validity of a limiting process. Other writers [2] have obtained the same results under the weaker hypothesis that the Cauchy data are $C^{(1)}[\lambda]$ on the line $x=\lambda, y=-\lambda$.

But the result on uniqueness is conditional on the existence and continuity of the derivatives (2). This involves a condition that is awkward to express for the derived uniqueness theorems for more general equations. In fact these theorems as stated in [2], [3] are not proven. To make this point clear let us consider Monge's equation

$$
A r+2 H s+B t=C .
$$

Here

$$
r=\frac{\partial^{2} z}{\partial x^{2}}, \quad s=\frac{\partial^{2} z}{\partial x \partial y}, \quad t=\frac{\partial^{2} z}{\partial y^{2}}
$$

We write also

$$
p=\frac{\partial z}{\partial x}, \quad q=\frac{\partial z}{\partial y} .
$$

In (3) the coefficients $A, H, \cdots$ will depend on $x, y, z, p, q$, and we suppose them to be functions of class $C^{(2)}$.

Five $C^{(1)}$ functions $x(\lambda), y(\lambda), z(\lambda), p(\lambda), q(\lambda)$ form a "strip" if

$$
d z=p d x+q d y
$$

identically in $\lambda$. Cauchy's problem for the equation (3) is to find a solution $z(x, y)$ of class $C^{(2)}$ which "contains" this strip. For equation (3), the strip is "regular" if

$$
A d y^{2}-2 H d x d y+B d x^{2} \neq 0
$$

and is "hyperbolic" if

$$
H^{2}-A B>0 .
$$

Set $w=H+\sqrt{H^{2}-A B}$; we may suppose that the square root is chosen so that $w$ does not vanish on the strip. Then

$$
\begin{gathered}
A B+w^{2}=2 H w \\
\left(w^{2}-A B\right)^{2}=4 w^{2}\left(H^{2}-A B\right)>0 .
\end{gathered}
$$

Lewy's characteristic equations belonging to the equation (3) may be written down in the form

$$
\begin{array}{ll}
w x_{\alpha}-A y_{\alpha}=0, & w y_{\beta}-B x_{\beta}=0, \\
w p_{\alpha}+B q_{\alpha}-C y_{\alpha}=0, & A p_{\beta}+w q_{\beta}-C x_{\beta}=0, \\
z_{\alpha}-p x_{\alpha}-q y_{\alpha}=0, & z_{\beta}-p x_{\beta}-q y_{\beta}=0
\end{array}
$$


where the suffixes denote partial derivatives:

$$
x_{\alpha}=\frac{\partial x}{\partial \alpha}, \text { etc. }
$$

This system of equations may be discussed in just the same.way as the pair (1). Under the assumptions indicated the coefficients in (9) are $C^{(2)}[x, y, z$, $p, q]$. It has been shown [2] that the system of equations (9) have a unique solution $x(\alpha, \beta), \cdots, q(\alpha, \beta)$ of class $C^{(1)}[\alpha, \beta]$ which reduce to $x(\lambda), \cdots, q(\lambda)$ on $\alpha=\lambda, \beta=-\lambda$, and for which all the derivatives

$$
x_{\alpha \beta}, \cdots, q_{\alpha \beta}
$$

exist and are continuous. The uniqueness established depends upon this last condition (10). If for instance the system (9) were satisfied by functions of class $C^{(1)}[\alpha, \beta]$ which satisfy also the conditions on $\alpha=\lambda, \beta=-\lambda$, but for which the derivatives (10) do not exist, then these functions would yield a solution of the regular hyperbolic Cauchy problem different from the one whose existence has been established. This possibility is not excluded by the discussion of $[1],[2],[3]$. Lewy recognises this effectively in a footnote to his paper.

The proof of the statement just made may be had in a few lines. If $\dot{x}=d x / d \lambda$, etc., we have

$$
\dot{x}=x_{\alpha}-x_{\beta}, \quad \dot{y}=y_{\alpha}-y_{\beta} .
$$

On $\alpha+\beta=0$, using (9),

$$
\begin{aligned}
\frac{\partial(x, y)}{\partial(\alpha, \beta)} \cdot\left(w^{2}-A B\right) & =\left|\begin{array}{ll}
x_{\alpha} & y_{\alpha} \\
x_{\beta} & y_{\beta}
\end{array}\right| \cdot\left|\begin{array}{cc}
w & -B \\
-A & w
\end{array}\right|=\left|\begin{array}{lr}
0,-B \dot{x}+w \dot{y} \\
w \dot{x}-A \dot{y}, & 0
\end{array}\right| \\
& =w\left(A \dot{y}^{2}-2 H \dot{x} \dot{y}+B \dot{x}^{2}\right) .
\end{aligned}
$$

Hence by (8), (5)

$$
\frac{\partial(x, y)}{\partial(\alpha, \beta)}=\frac{A \dot{y}^{2}-2 H \dot{x} \dot{y}+B \dot{x}^{2}}{2 \sqrt{ }\left(H^{2}-A B\right)} \neq 0 .
$$

This implies also $y_{\alpha} \neq 0$. We may therefore express $z, p, q$ as functions of $x, y$ of class $C^{(1)}$. Then

$$
d z=z_{\alpha} d \alpha+z_{\beta} d \beta=\left(p x_{\alpha}+q y_{\alpha}\right) d \alpha+\left(p x_{\beta}+q y_{\beta}\right) d \beta=p d x+q d y .
$$

So $p=\partial z / \partial x, q=\partial z / \partial y$ and $z$ is of class $C^{(2)}[x, y]$. Finally, from equation (9),

$$
\begin{aligned}
0 & =w^{2} p_{\alpha}+w B q_{\alpha}-w C y_{\alpha} \\
& =w^{2}\left(r x_{\alpha}+s y_{\alpha}\right)+w B\left(s x_{\alpha}+t y_{\alpha}\right)-w C y_{\alpha} \\
& =A w y_{\alpha} r+\left(w^{2}+A B\right) y_{\alpha} s+B w y_{\alpha} t-C w y_{\alpha} \\
& =w y_{\alpha}[A r+2 H s+B t-C] .
\end{aligned}
$$

Since $w y_{\alpha} \neq 0$, so equation (3) is satisfied. 
It will be seen that, contrary to the statements of the text books, we cannot infer on the basis of anything yet proved, the uniqueness of the solution of Cauchy's problem for functions $z(x, y)$ of class $C^{(2)}$ simply. To obtain any statement of uniqueness for equation (3) we would need to admit only those solutions of class $C^{(2)}$ such that, when $x, y, z, p, q$ are expressed by means of the characteristic parameters $\alpha, \beta$, all the mixed derivatives (10) exist and are continuous. This is the awkward and unsatisfactory result for Monge's equation which I referred to at the beginning. It wil be clear also that what we need is an unconditional uniqueness theorem; in the case of the pair of equations ( 1 ) we need a proof of uniqueness which is independent of the existence or otherwise of the derivatives (2). Such a proof will now be given.

\section{The Uniqueness Theorem}

Suppose $z_{1}, z_{2}$ and $\bar{z}_{1}, \bar{z}_{2}$ are two solution-pairs of (1) of class $C^{(1)}[x, y]$ and that on $x+y=0$,

$$
z_{1}=\bar{z}_{1}, \quad z_{2}=\bar{z}_{2} .
$$

Write $\bar{a}_{i j}=a_{i j}\left(\bar{z}_{1}, \bar{z}_{2}\right)$. Set $u_{1}=z_{1}-\bar{z}_{1}, u_{2}=z_{2}-\bar{z}_{2}$ so that $u_{1}, u_{2}$ both vanish on $x+y=0$. Now we find

$$
\begin{aligned}
& \frac{\partial}{\partial x}\left(a_{11} u_{1}\right)=a_{11} \frac{\partial z_{1}}{\partial x}-\bar{a}_{11} \frac{\partial \bar{z}_{1}}{\partial x}+u_{1} \frac{\partial}{\partial x}\left(a_{11}\right)+\left(\bar{a}_{11}-a_{11}\right) \frac{\partial \bar{z}_{1}}{\partial x}, \\
& \frac{\partial}{\partial x}\left(a_{12} u_{2}\right)=a_{12} \frac{\partial z_{2}}{\partial x}-\bar{a}_{12} \frac{\partial \bar{z}_{2}}{\partial x}+u_{2} \frac{\partial}{\partial x}\left(a_{12}\right)+\left(\bar{a}_{12}-a_{12}\right) \frac{\partial \bar{z}_{2}}{\partial x} .
\end{aligned}
$$

Adding, and using equations (1),

$$
\begin{aligned}
\frac{\partial}{\partial x}\left(a_{11} u_{1}+a_{12} u_{2}\right)= & u_{1} \frac{\partial}{\partial x}\left(a_{11}\right)+u_{2} \frac{\partial}{\partial x}\left(a_{12}\right)+\left(\bar{a}_{11}-a_{11}\right) \frac{\partial \bar{z}_{1}}{\partial x} \\
& +\left(\bar{a}_{12}-a_{12}\right) \frac{\partial \bar{z}_{2}}{\partial x} .
\end{aligned}
$$

Now apply the mean value theorem to the differences $\bar{a}_{11}-a_{11}, \bar{a}_{12}-a_{12}$. Then we can find a constant $K_{1}>0$ such that near $x=0, y=0$

$$
\left|\frac{\partial}{\partial x}\left(a_{11} u_{1}+a_{12} u_{2}\right)\right| \leqq K_{1}\left(\left|u_{1}\right|+\left|u_{2}\right|\right)
$$

In just the same way,

$$
\left|\frac{\partial}{\partial y}\left(a_{21} u_{1}+a_{22} u_{2}\right)\right| \leqq K_{1}\left(\left|u_{1}\right|+\left|u_{2}\right|\right) .
$$

Again, if

$$
u=a_{11} u_{1}+a_{12} u_{2}, \quad v=a_{21} u_{1}+a_{22} u_{2}
$$


we find, for a suitable constant $K_{2}$,

$$
\left.\begin{array}{l}
\left|u_{1}\right|=\left|\frac{1}{\Delta}\left(a_{22} u-a_{12} v\right\rangle\right| \leqq K_{2}(|u|+|v|) \\
\left|u_{2}\right|=\left|\frac{1}{\Delta}\left(a_{11} v-a_{21} u\right)\right| \leqq K_{2}(|u|+|v|)
\end{array}\right\} .
$$

Hence if $K=2 K_{1} K_{2}$,

$$
\left|\frac{\partial u}{\partial x}\right| \leqq K(|u|+|v|),\left|\frac{\partial v}{\partial y}\right| \leqq K(|u|+|v|) .
$$

From these inequalities (12) and the fact that $u=0, v=0$ on $x+y=0$ it follows that $u, v$ vanish identically. For, choose a constant $G$ such that

$$
|u| \leqq G, \quad|v| \leqq G .
$$

Considering the part of the plane $x+y>0$, set

$$
\zeta_{n}=\frac{G}{n !}(2 K)^{n}(x+y)^{n}
$$

so that

$$
\frac{\partial \zeta_{n}}{\partial x}=\frac{\partial \zeta_{n}}{\partial y}=2 K \zeta_{n-1}
$$

By induction we find, in $(x+y)>0$,

$$
|u| \leqq \zeta_{n}, \quad|v| \leqq \zeta_{n} .
$$

This is true already for $n=0$; if we suppose it holds for $(n-1)$ then

$$
\begin{gathered}
|u|=\left|\int_{-y}^{x} \frac{\partial u}{\partial x} d x\right| \leqq \int_{-y}^{x} 2 K \zeta_{n-1} d x \\
=\int_{-y}^{x} \frac{\partial \zeta_{n}}{\partial x} d x=\zeta_{n}
\end{gathered}
$$

and similarly $|v| \leqq \zeta_{n}$.

Since $\zeta_{n} \rightarrow 0$ as $n \rightarrow \infty$, so we infer $u=0, v=0$, in $x+y>0$. It is obvious that the result holds also for $x+y<0$.

Now from (11), $u_{1}, u_{2}$ vanish identically, so that $z_{1}=\bar{z}_{1}, z_{2}=\bar{z}_{2}$, and any $C^{(1)}$ solution of the Cauchy problem for (1) is unique. Of course, it now follows from the existence theorem itself, that a $C^{(1)}$ solution necessarily admits the continuous derivatives (2).

It will be clear that the discussion above extends so as to obtain similar results for a system of $N=m+n$ equations of the form

$$
\begin{aligned}
& \sum_{j=1}^{N} a_{i j} \frac{\partial z_{j}}{\partial x}=0, \quad i=1,2, \cdots, m . \\
& \sum_{j=1}^{N} a_{i j} \frac{\partial z_{j}}{\partial y}=0, \quad i=m+1, \cdots, N .
\end{aligned}
$$




\section{References}

[1] H. Lewy: Math. Ann. Band 98, 1927.

[2] Courant u. Hilbert: Methoden der mathematischen Physik, Band II, Kap. 5. Berlin: Springer 1937.

[3] R. Sauer: Anfangswertprobleme bei partiellen Differenzialgleichungen, Kap. 3. Berlin: Springer 1958.

University of Sydney. 